\title{
On the Selection of Radiating Elements for Compact Indoor Massive-Multiple Input Multiple Output Base Stations
}

Xavier Artiga, Bertrand Devillers, and Julien Perruisseau-Carrier

This paper is a postprint of a paper submitted to and accepted for publication in IET Microwaves, Antennas \& Propagation and is subject to Institution of Engineering and Technology Copyright. Thecopy of record is available at IET Digital Library 


\title{
On the Selection of Radiating Elements for Compact Indoor Massive-MIMO Base Stations
}

\author{
Xavier Artiga, Bertrand Devillers, and Julien Perruisseau-Carrier
}

\begin{abstract}
Massive-MIMO was recently proposed as a key solution for improving the performance of multiuser MIMO in future cellular generations. The main paradigm in this novel MIMO technique is to use a very large number of antenna elements at the base station (BS), which was shown to drastically increase capacity while saving transmission power. However, envisioning indoor BS with such number of antennas it is essential to assess the implementation of compact arrays based on different antenna candidates, which constitutes the main objective of this work. Antenna solutions ranging from miniaturized patches to triple-polarized radiators are proposed. Characteristics such as return loss and mutual couplings are obtained via full-wave electromagnetic simulations and as a function of elements density in a given limited BS real estate. The different antenna candidates are compared by computing the signal to interference plus noise ratio in both noise and interference-limited regimes and for different multi-user MIMO precoding strategies. This analysis allows reporting on optimal antenna densities above which performance degrades despite an increasing number of antennas, and identifying the main cause of this limitation for the different antenna types and scenarios. Finally, practical considerations such as antenna miniaturization, bandwidth, and cost, are included in the discussion.
\end{abstract}

\section{INTRODUCTION}

Following years of intense research and development, MIMO technology has finally proved to effectively fulfill its promises in "real world", and is now being introduced in the latest wireless

\footnotetext{
Manuscript received February 1st, 2012. This work was supported by the European Commission through the BuNGee project (ICT-FP7-248267) and COST action VISTA IC1102, and by the Swiss National Science Foundation (SNSF) under grant n02133583.

X. Artiga and B. Devillers are with the Centre Tecnològic de Telecomunicacions de Catalunya (CTTC), Castelldefels, Barcelona, Spain.

J. Perruisseau-Carrier is with the Adaptive MicroNanoWave Systems Group, LEMA/Nanolab, Ecole Polytechnique Fédérale de Lausanne (EPFL), Lausanne, CH-1015, Switzerland.
} 
standards such as WiMAX and LTE-A. Nevertheless, to approach the spectral efficiency achievable in theory, point-to-point MIMO requires complex multi-antenna user terminals and nonline-of-sight (NLOS) propagation environments with rich scattering [1]-[4]. These shortcomings have been addressed by the development of multi-user MIMO techniques, which allow the use of single-antenna user terminals while being more robust to poor scattering or line-of-sight (LOS), thanks to the large distance between the different terminals [5]-[7].

Targeting a drastic performance improvement for future cellular generations, an extremely promising multi-user MIMO technology has been recently proposed [8]-[12]. It consists of using base stations (BSs) having a number of antennas well above the number of active users, which in practice would mean having BSs with hundreds of antennas. This huge number of antennas allows simultaneously sending independent data to the different users with very narrow beams, which leads to an unprecedented capacity increase and transmission power save [12]. This new class of multi-user MIMO has been referred to as Massive-MIMO in [10] and very large MIMO in [11]. We will use Massive-MIMO in the remainder of this paper.

Foundational work on Massive-MIMO demonstrated that the effects of uncorrelated noise and fast fading vanish when the number of antennas increases without limit [8]. In these conditions, simple linear precoders such as maximum ratio transmission [9] or zero forcer [10] where found to be near optimal solutions. However, the capacity scaling with the number of BS antennas predicted by these theoretic studies entails in practice many design challenges related with synchronization and channel estimation as well as cost or size. Some of them have been recently addressed in the first Massive-MIMO testbed [12], but a solution for accommodating very large number of antennas in constrained practical BS real estates taking in consideration electromagnetic coupling between antennas is still missing. Such compact arrays can become essential when indoor pico- or femto-cell base stations are envisioned.

The effects of the electromagnetic interaction between antennas at each end of a MIMO link have been studied extensively in most scenarios of interest so far [13]-[16]. It is well-known that a first consequence of mutual coupling is the reduction of the power transmitted/received by the antenna array, which translates into lower array efficiency. This degradation is caused by the variation of the individual antennas input impedance, which increases return loss, and by the power dissipated in the loads of surrounding array elements. The second well-known effect of mutual coupling is its contribution to MIMO signal correlations. 
The consequence of these antenna limitations on MIMO performance have been studied in [17], [18] by evaluating the effect of packing an increasing number of antennas in a given physical space, in the single-user case. It was shown that the ergodic MIMO capacity monotonically increases with the number of antennas when only signal correlation is considered, whereas an optimum number of antennas is found when both correlation and array efficiency are accounted for. Only uniform linear arrays of half wavelength dipoles in free space were evaluated since analytic mutual coupling expressions are available in this case.

In this work, we address the implementation of compact antenna arrays for indoor MassiveMIMO base stations. In particular, we provide a simple method for the selection of an optimum radiating element taking in account the effects of correlation and mutual coupling. Antenna coupling in Massive-MIMO has also been recently discussed in [11], even though it was not the main focus of the study. Moreover, for the reason mentioned in the previous paragraph, again only half wavelength dipoles array in free space were evaluated using an analytic formulation. Here different antenna solutions, ranging from miniaturized patches to triple-polarized radiators, are evaluated and compared using the two above-mentioned multi-user MIMO precoders (maximum ratio transmission and zero-forcer). Mutual coupling is determined by electromagnetic full-wave simulations, which constitutes an accurate and general approach that can be extended to any real-world BS antenna element.

Notation: Boldface uppercase letters denote matrices and boldface lowercase letters refer to column vectors. We denote by $(.)^{*}$ and $(.)^{H}$ the conjugate and Hermitian transpose, respectively. The $N \times N$ identity matrix is denoted by $\mathbf{I}_{N}$, and $\operatorname{trace}(\mathbf{A})$ refers to the trace of matrix $\mathbf{A}$. Finally, nonboldface uppercase letters are used to refer to the entries of a matrix: the $(k, l)$ th entry of the matrix $\mathbf{W}$ is denoted by $W_{k l}$.

\section{SySTEM MODEL}

\section{A. Multi-User MIMO System}

A single cell with a base station equipped with an array of $M$ antennas, and $K$ single antenna user terminals is considered. We are interested in analyzing the evolution of the system performance as the number of antennas $M$ grows without limit, for fixed number of user $K$. The focus is set on the downlink transmission, for which the multi-user MIMO system model 
can be written as

$$
\mathbf{y}=\mathrm{Gs}+\mathbf{w}
$$

where $\mathbf{y}$ is a $K \times 1$ received vector containing the stack of the received signals at all user terminals. Similarly, the $K \times 1$ vector $\mathbf{w}$ denotes the stack of all received noise components, which are modeled as independent and identically distributed (i.i.d.) complex circular symmetric Gaussian random variables with zero mean and variance $\sigma_{w}^{2}$. The channel matrix $\mathbf{G}$ is of size $K \times M$. Its component $G_{k m}$ refers to the channel gain from the $m^{\text {th }}$ transmit antenna to user terminal $k$. The $M \times 1$ transmit signals vector $\mathbf{s}$ has to comply with a transmit power constraint so that

$$
E\left[\mathbf{s}^{H} \mathbf{s}\right] \leq P,
$$

where $P$ denotes the total transmit power, whose value is independent of the number of transmit antennas. The constraint (2) thus ensures a fair comparison between scenarios with different number of transmit antennas. The ratio between the transmit power and noise variance is denoted by $\rho=P / \sigma_{w}^{2}$.

We assume linear precoding at the base station, such that the transmitted vector is rewritten as

$$
\mathrm{s}=\mathbf{F a}
$$

where the $K \times 1$ vector a contains the stack of the $K$ information symbols intended for the $K$ user terminals. Independent unit energy constellation symbols are assumed, i.e. $E\left[\mathbf{a a}^{H}\right]=\mathbf{I}_{K}$, such that the power constraint can be rewritten in terms of the $M \times K$ precoding matrix $\mathbf{F}$ as

$$
\operatorname{trace}\left(\mathbf{F} \mathbf{F}^{H}\right) \leq P
$$

In this paper, two versions of linear precoding are considered and described next.

1) Zero-Forcing Linear Precoding: The zero-forcing (ZF) criterion aims at a complete cancellation of the inter-user interference. The corresponding precoder is a scaled version of the pseudoinverse of the channel matrix

$$
\mathbf{F}=\sqrt{\frac{P}{\operatorname{trace}\left(\left(\mathbf{G G}^{H}\right)^{-1}\right)}} \mathbf{G}^{H}\left(\mathbf{G G}^{H}\right)^{-1},
$$


where the scaling factor (inside the square root sign) is such as to satisfy (4). This precoder equalizes the signal to noise ratio (SNR) among users. The resulting SNR is

$$
S N R=\frac{P}{\sigma_{w}^{2} \operatorname{trace}\left(\left(\mathbf{G G}^{H}\right)^{-1}\right)}=\frac{\rho}{\operatorname{trace}\left(\left(\mathbf{G G}^{H}\right)^{-1}\right)}
$$

2) Maximum Ratio Transmission: Besides the zero-forcing precoder, we consider the less demanding maximum ratio transmission (MRT), which corresponds to the following precoder

$$
\mathbf{F}=\sqrt{\frac{P}{\operatorname{trace}\left(\mathbf{G G}^{H}\right)}} \mathbf{G}^{H}
$$

which, again, complies with (4). Denoting $\mathbf{A}=\frac{1}{M} \mathbf{G G}^{H}$, the $k$ th user signal to interference plus noise ratio (SINR) associated with precoder (7) can be calculated as

$$
S I N R_{k}=\frac{\rho A_{k k}^{2}}{\frac{\operatorname{trace}(\mathbf{A})}{M}+\rho \sum_{l=1, l \neq k}^{K}\left|A_{k l}\right|^{2}}
$$

\section{B. Mutual coupling and channel model}

The simple Kronecker model [19] is employed, which assumes that the random fading processes at the receiver are uncorrelated to those at the transmitter. Though several studies demonstrated some of its deficiencies, its simplicity makes the Kronecker model be a good starting point in the analysis of MIMO systems [19] and was considered a good option for the purpose of this work, namely the analysis and comparison of different antenna solutions for Massive-MIMO. Moreover, this model is quite efficient for indoor scenarios with uniform angle of arrival/departure as assumed later. It is also noticeable that this model was also used in [11], [17], [18].

The Kronecker channel matrix can be expressed as

$$
\mathbf{G}=\mathbf{R}_{R}^{1 / 2} \mathbf{H}\left(\mathbf{R}_{T}^{1 / 2}\right)^{T}
$$

where $\mathbf{R}_{R} \in \mathbb{C}^{K \times K}$ and $\mathbf{R}_{T} \in \mathbb{C}^{M \times M}$ are the receive and transmit signal correlation matrices respectively and $\mathbf{H} \in \mathbb{C}^{K \times M}$ is a random matrix with all the elements being i.i.d. zero mean complex circular symmetric Gaussian random variables with unit variance. The antennas on the users side are uncorrelated and uncoupled due to the very large distance between them (there is only one antenna per user), hence $\mathbf{R}_{R}=\mathbf{I}_{K}$. The BS correlation matrix can be then calculated from [19]

$$
R_{T_{i j}}=\frac{X_{T_{i j}}}{\sqrt{X_{T_{i i}} X_{T_{j j}}}}
$$


where $\mathbf{X}_{T} \in \mathbb{C}^{M \times M}$ is the covariance matrix of the transmit signals. For the indoor environment we assume an ideally scattered environment in which: (i) the outgoing waves from the BS are uniformly distributed over space, i.e. uniform 3D angular power spectrum (uniform angular distributions of clusters of scatterers were observed in [20]; and (ii)the cross-polarization ratio (XPR) is close to $0 \mathrm{~dB}[21]$. Under these conditions, the covariance matrix can be calculated as [19]

$$
\mathbf{X}_{T}=C\left(\mathbf{I}_{M}-\mathbf{S}_{T} \mathbf{S}_{T}^{H}\right)
$$

where $\mathbf{S}_{T}$ is the $\mathbf{S}$-parameters matrix of the transmit antenna array and $\mathrm{C}$ is a constant that depends on the radiated power [19]. It is also worth underlining that (11) neglects thermal losses in the radiators themselves (dielectric and conductor loss), which is a valid assumption since in Massive-MIMO efficiency loss due to power coupled to surrounding elements loads will largely exceed thermal losses in the radiators.

The power coupled between each pair of antennas in the array (i.e. mutual coupling) is directly accounted for in the non-diagonal terms of $\mathbf{S}_{T}$. In turn, the transmitted power reduction caused by mutual coupling is accounted for in the diagonal-terms of $\mathbf{X}_{T}$ in (11) [19]. However, this effect is normalized out in (10). Therefore, we use (9)-(11) to evaluate the contribution of the signal correlation only and (11)-(12) to take in account both transmitted power reduction and signal correlation.

$$
\mathbf{G}=\mathbf{H}\left(\mathbf{X}_{T}^{1 / 2}\right)^{T}
$$

It is also worth mentioning that for lossless antennas in uniform 3D angular power spectrum (APS) scenarios, signal correlation is caused by mutual coupling only so the transmitted power reduction and signal correlation are directly related. Therefore it only makes sense to evaluate the correlation effects alone in the case where there is no restriction in the transmitted power or of arbitrary low noise receivers, i.e. in high SNR scenarios.

Though for simplicity here we only consider ideally scattered channels, more complex scenarios with non-uniform angular power distributions can be evaluated using a more detailed formulation for the covariance matrix. In this case, the matrix is a function of the radiation patterns of each antenna and of the outgoing waves APS distribution [19]. In a similar way, scenarios with higher XPR can be analyzed following the procedure in [22]. 


\section{Antenna Solutions And Results}

\section{A. Content and Methods}

This section evaluates the impact of mutual coupling on the performance of an indoor MassiveMIMO downlink through the averaged SINR per user. Different BS antenna elements, ranging from simple dipoles to tri-polarized antennas, are proposed and their performance assessed in detail under the use of two linear precoding schemes (zero-forcer and the maximum ratio transmission). Two different operation regimes are also considered: an interference-limited regime (characterized by a high average $\operatorname{SNR} \rho=20 \mathrm{~dB}$ ), and a noise-limited regime (associated with a low average $\mathrm{SNR} \rho=0 \mathrm{~dB}$ ). Note that we assume that there is no external source of interference than that caused by the multiple streams sent to multiple users. Finally, since mutual coupling depends on the loads at the different antenna ports, different matching networks are discussed.

Expressions (6) and (8) are used to compute the average SINR per user. As detailed in Section II-B, the different effects of mutual coupling (correlation and efficiency loss) can be artificially isolated using different expressions for the transmit antenna correlation matrix. The number of users is set to $K=4$ and for each test case the SINR values obtained with 500 realizations of the channel matrix $\mathbf{G}$ are averaged. The finite-elements Ansys HFSS full-wave solver was used to obtain the S-parameters matrices of the transmit antenna array, needed for the calculation of the correlation matrices.

First, different Massive-MIMO BS candidate antennas were designed for a $50 \Omega$ reference impedance in isolation at $2.5 \mathrm{GHz}$ (note that in the case of multi-polarized elements we actually refer here to a "unit" multi-port element of the array, which actually constitute several antennas from the channel perspective). Then, for each antenna type several 2D square-lattice arrays with an increasing number of antennas per surface unit (referred to as here as the antenna "density") was computed. The entire analysis is carried out at a single frequency of $2.5 \mathrm{GHz}$ in order to save computation time, so the conclusions derived from the comparison of different elements are only valid if all elements satisfy the bandwidth requirements of the communications system.

The electrical size of the simulated arrays ranges from $\lambda \times \lambda$ to $2 \lambda \times 2 \lambda$. This size is limited by the memory used by the full-wave solver - some of our simulations required up to 16 Gbits of RAM memory- but it was shown that similar SINR results are obtained for different array sizes within this range (after appropriate scaling by the total number of antenna). Therefore, 
simulations that can be run on standard modern PC are sufficient to assess the performance of arbitrary large arrays. In turn, we present the SINR per surface unit $\left(\lambda^{2}\right)$ as a function of the antenna density (\# antennas $/ \lambda^{2}$ ), so as to assess and compare solutions independently of the global BS size.

\section{B. BS antenna candidates}

Half-wavelength dipoles, single and dual-polarized microstrip patches and tri-polarized radiators are proposed as BS antenna elements. Half-wavelength are considered to be vertically oriented and arranged in a 2D grid in the horizontal plane. Such elements are of limited interest in practice since they cannot be easily integrated with supporting structures and RF circuitry, due to the absence of a ground plane. Moreover the ground plane also provides isolation between the antennas, RF circuitry, and allows guaranteeing performance roughly independent of the location of the BS (which could be placed on a post or a wall). However they are included here because the fact that they can be packed in very small lattices allows making important observations. Quarter-wavelength monopoles can be used to overcome the absence of ground plane, though here we consider the use of coaxial fed microstrip patches because of their low profile which would allow an seamless integration of compact arrays in walls or roofs. Dual polarized patches are also studied since it is well-known that using dual-polarized antennas enable additional MIMO channels, and in turn MIMO gain with respect to the single-polarized case. Polarization diversity can be further exploited using the third orthogonal orientation of the electric field (e.g. vertical polarization if we consider a horizontal patch). Here this additional dimension is used by inserting a vertical monopole at the center of the patch as depicted in Fig. 1(a). Low-profile tri-polarized solutions can be also implemented [23] though they are not considered here in order to simplify simplifying the computation of large arrays.

Table I summarizes the main characteristics of the designed elements. The thermal loss efficiency of all studied elements was shown to be better than $95 \%$, which validates the corresponding assumption made in Section II-B. For the microstrip elements, a $1.57 \mathrm{~mm}$-thick substrate with $\epsilon_{r}=2.2$ was used. The main drawback of microstrip patches over rather thin substrates is their narrowband operation. Though the designed patch can cover the $20 \mathrm{MHz}$ channels bandwidths of actual wireless standards such as TDD-LTE [24], in practice BS antennas may require much larger

bandwidths depending on the licenses acquired by a given operator. Therefore, in Section III-E 
TABLE I. ANTENNA SOLUTIONS OVERVIEW

\begin{tabular}{|c|c|c|c|}
\hline $\begin{array}{c}\text { Antenna } \\
\text { element }\end{array}$ & $\begin{array}{c}\text { Lateral size } \\
{[\mathbf{m m}]}\end{array}$ & $\begin{array}{c}\text { BW (6dB) } \\
\text { (worst case) } \\
{[\mathbf{M H Z}]}\end{array}$ & $\begin{array}{c}\text { Isolation } \\
\text { (worst case) } \\
\text { [dB] }\end{array}$ \\
\hline Dipole & 2 & 415 & NA \\
\hline Single-pol. patch & 38.7 & 58 & NA \\
\hline Dual-pol. patch & 38.7 & 58 & 51.8 \\
\hline $\begin{array}{c}\text { Tri-pol. with } \\
\text { monopole }\end{array}$ & 38.6 & 60 & 20.6 \\
\hline $\begin{array}{c}\text { Miniaturize } \\
\text { dual-pol. patch }\end{array}$ & 25.5 & 32 & 21.6 \\
\hline $\begin{array}{c}\text { Thick substrate } \\
\text { Single-pol. patch }\end{array}$ & 46.5 & 225 & NA \\
\hline $\begin{array}{c}\text { Thick substrate } \\
\text { dual-pol. patch }\end{array}$ & 46 & 225 & 25 \\
\hline $\begin{array}{c}\text { Stacked single } \\
\text {-pol. patch }\end{array}$ & 38.3 & 180 & NA \\
\hline $\begin{array}{c}\text { Stacked dual } \\
\text {-pol. patch }\end{array}$ & 38.3 & 180 & 24 \\
\hline
\end{tabular}

we present successful techniques for increasing the bandwidth of such planar patch elements in the Massive-MIMO context.

For the sake of completeness miniaturization and decoupling structures are also briefly discussed later in Section III-F .

\section{Results}

Fig. 2 and Fig. 3 show a comparison of the averaged SINR per user obtained using the different radiating element candidates for the two precoding schemes and the two SNR operation regimes considering (i) only correlation and (ii) correlation and efficiency. These to sets of curves are obtained using the input impedance single-port match which provides an improved performance with respect other known single port matching techniques as discussed in Section III-D. The ideal case of uncoupled and uncorrelated antennas (i.e. $\mathbf{S}_{T}=0$ and $\mathbf{R}_{T}=\mathbf{I}_{M}$ ) is also included in the figures as a reference. 

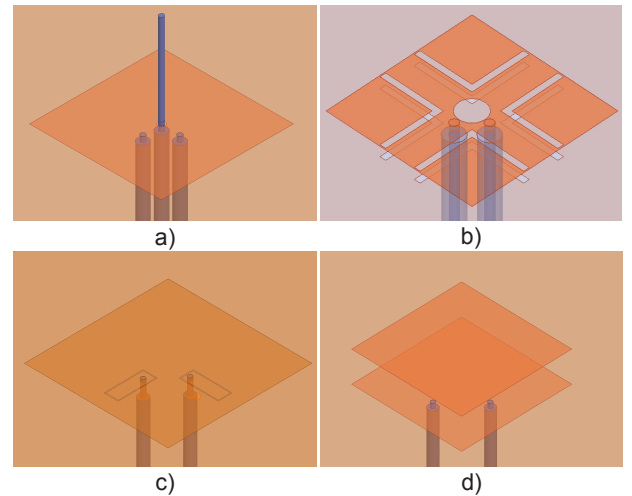

Fig. 1. Main antenna elements proposed and evaluated for Massive-MIMO BS. a) Tri-polarized antenna based on an horizontal dual-polarized patch and a vertical monopole. b) Miniaturized dual-polarized patch. c) Improved-bandwidth microstrip patch using a thick substrate. d) Improved-bandwidth patch using stacked patches.

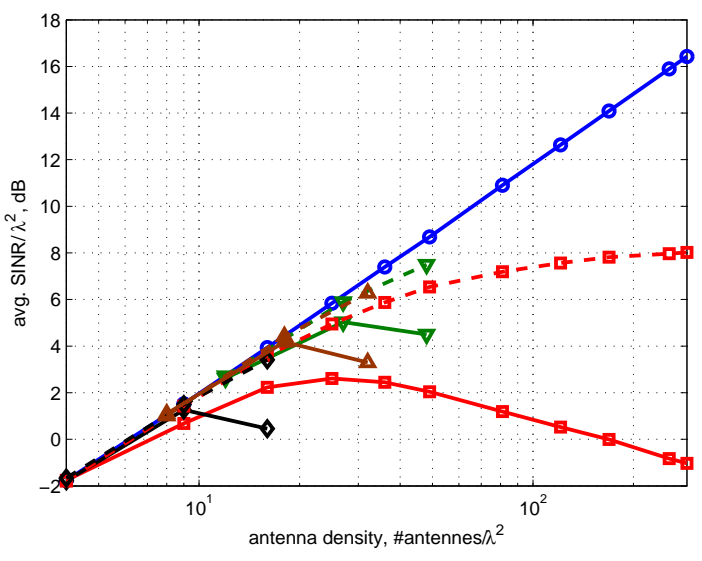

(a) noise-limited regime $\rho=0 d B$

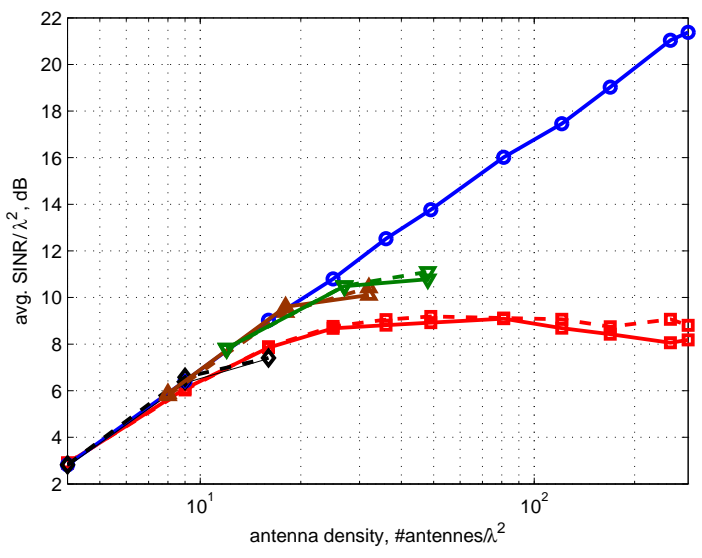

(b) interference-limited regime $\rho=20 d B$

Fig. 2. Average SINR as a function of antenna density for the MRT precoder. (-):correlation and efficiency ( - - ):correlation only(○): ideal antennas $(\square)$ : dipoles $(\diamond)$ : single-polarized patches $(\triangle)$ : dual-polarized patches $(\nabla)$ : tri-polarized radiators

1) Radiating elements comparison: As expected, in all the considered scenarios (precoding technique-SNR operating regime) uncoupled and uncorrelated antennas lead to a monotonically increasing SINR. However, the theoretic performance improvement due to the increasing number of antennas is not achieved when mutual coupling is accounted for. In fact, an optimum point 


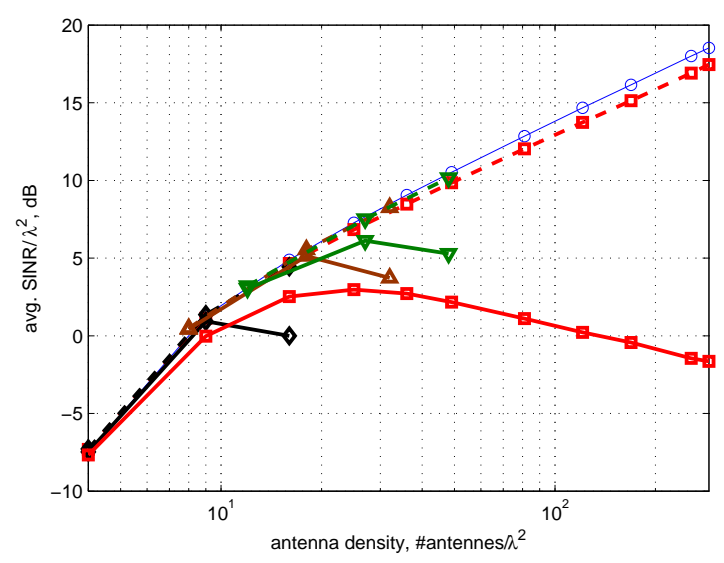

(a) noise-limited regime $\rho=0 d B$

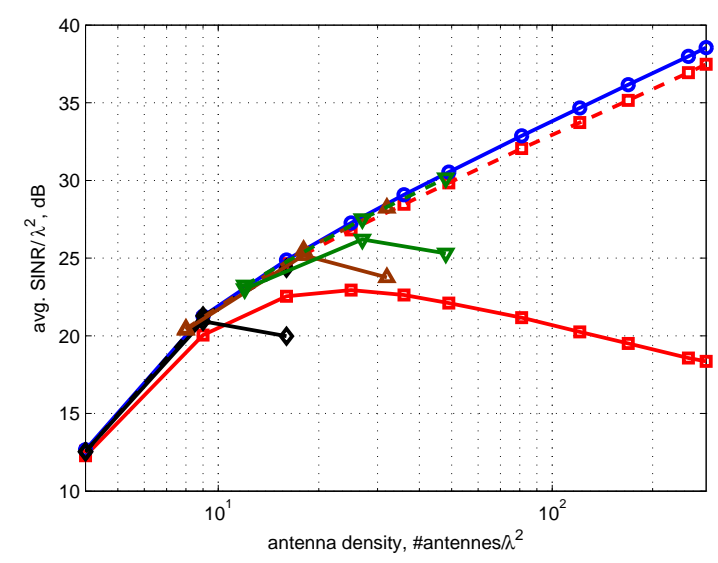

(b) interference-limited regime $\rho=20 d B$

Fig. 3. Average SINR as a function of antenna density for the ZF precoder. (-):correlation and efficiency ( - - ):correlation only(○): ideal antennas $(\square)$ : dipoles $(\diamond)$ : single-polarized patches $(\triangle)$ : dual-polarized patches $(\nabla)$ : tri-polarized radiators

(from which increasing the antenna density reduces the MIMO performance) is found regardless of the radiating element or the scenario. For each radiating element this optimum antenna density is the same in the four considered scenarios, namely 25 antennas $/ \lambda^{2}$ (which corresponds to an element spacing of $\lambda / 4$ ) for dipoles; and 9, 18 and 27 antennas $/ \lambda^{2}$ (which corresponds to an element spacing of $\lambda / 2$ from patch center to patch center) for single-, dual, and tri-polarized patches respectively. Therefore the general qualitative performance limitation of compact indoor Massive-MIMO BS due to antenna coupling is quite independent of the type of antennas or precoding scheme used, as long as sufficient coupling between elements is reached for increased densities. It must be noted that, for the MRT in the interference-limited regime (see Fig. 2(a)), increasing the antenna density beyond the optimum point does not reduce the average SINR but only provides very marginal improvements. It is also worth mentioning that the sharp SINR decrease obtained beyond the optimum point for patch based elements (especially in noise-limited scenarios) is due to the rather high lateral size of the elements. At the maximum density, the elements are almost touching each other since the inter-element spacing (i.e. $\lambda / 3$ from patch center to patch center) is very close to the element's lateral size. So this produces a sharp increase of the mutual coupling from the optimum point to the maximum density point that leads to the sharp SINR transition.

Then the main objective of antenna designers in indoor Massive-MIMO is to compact as 
many uncoupled antennas as possible in realistic array sizes. It is observed that polarization diversity works in this direction since it allows larger antenna densities before mutual coupling effects cancel the benefit brought by additional antenna elements (note that, logically, a single tri-polarized element means three antennas in the definition of antenna density used here). This is beneficial to the MIMO performance since higher SINR are associated with the optimum achieved by enabling polarization diversity. The improvement achieved by dual-polarization ( $2.8 \mathrm{~dB}$ for MRT and noise-limited regime) is close to the theoretical value of $3 \mathrm{~dB}$, while it is slightly below for triple polarization $(3.8 \mathrm{~dB}$ vs $4.8 \mathrm{~dB})$. This is because the monopoles included in the tri-polarized radiator, in contrast with the patches, provide SINR below ideal even for small densities such as 9 antennas $/ \lambda^{2}$. This is also observed in the dipole case.

Therefore, it can be concluded that in indoor massive-MIMO scenarios with XPR close to 0 $\mathrm{dB}$, the use of dual-polarized patches is a more effective way of compacting antennas than using dipoles, despite the fact that dipoles allow extremely small inter-element spacing. Exploiting the third polarization by the use of a monopole provides further SINR improvement, but with a non-negligible degradation with respect to ideal uncoupled antennas. It must be recalled that the benefit of using tri-polarized elements reported here is subject to the existence of a XPR close to $0 \mathrm{~dB}$ between all three polarizations.

2) Array efficiency and antenna correlation effects: Fig. 2 and Fig. 3 also allow identifying the mutual coupling effect that limits the Massive-MIMO performance in each scenario. For the MRT, a clear optimum antenna density is found in the noise-limited regime when both correlation and efficiency are considered, whereas the SINR still monotonically increases with the density of antennas beyond the optimum, when only correlation is considered. Therefore, in the noise-limited regime, the loss of transmitted power constitutes the most limiting factor. In contrast, the results clearly show that in the interference-limited regime the reduction in the transmitted power has little impact on the performance, which is limited by the signal correlation at the antennas. This result was expected since the signal correlation is directly responsible for the multi-user interference, which dominates SINR in the interference-limited regime.

In contrast, the $\mathrm{ZF}$ is always limited by the array efficiency because of its well-known interference-canceling capability. It can be observed that the only correlation curves perform close to the ideal case since in the uniform 3D APS scenario considered here, the multiuser interference is only caused by signal correlation induced by mutual coupling. Due to the 
interference canceling property, in the high SNR regime the ZF clearly outperforms the MRT since an increase in the SNR translates in a direct improvement of the ZF performance, whereas both achieve similar performances in the noise-limited regime. Therefore, as observed in pointto-point MIMO and without taking in consideration the implementation complexity that can be a critical aspect [12], ZF becomes better solution than the MRT transmission scheme for indoor Massive-MIMO as the SNR grows.

\section{Impedance Matching Conditions}

The effect of mutual coupling on MIMO system performances depends on the loads at the different antenna ports, and thus the design of matching networks for MIMO antennas has been widely investigated in single-user MIMO. It was found that the multi-port conjugate match [25] is an optimum solution that can cancel out both correlation and return loss for uniform 3D APS. However, such a matching network is obviously not suitable for Massive-MIMO since it requires a new branch for each non-zero coupling term. Moreover, in addition to prohibitive complexity thermal loss would become an issue in such large networks. As a result, real Massive-MIMO implementations should only consider single-port matching solutions in which the matching networks have a number of independent branches equal to the number of antennas. In this section we compare (i) the characteristic impedance match, in which each antenna is terminated with the reference impedance namely $Z_{L}=Z_{0}$; (ii) the self-impedance match, where each antenna is terminated by a load equal to the conjugate of its self-impedance, namely $Z_{L}=Z_{11}^{*}$; and (iii) the input impedance match, in which each antenna is terminated by the conjugate of the input seen looking into each port, which includes both self- and mutual-impedances [25]. For single or uncoupled antennas the self-impedance match leads to maximum power transfer, but will only be approximately true when real coupling is considered. The input impedance match assures that no power is reflected in any port but it does not avoid the power from one antenna being coupled to another one. Other single-port matching solutions were proposed to optimize either capacity or transmit power [25]-[27], but in general only two-antenna systems were considered and their extension to a large number of antennas appears extremely complex.

Fig. 4 allows comparing the performance of the three different matching conditions for all the BS candidate elements and for the noise-limited regime and MRT scenario. The results for other scenarios are qualitatively similar and thus are omitted for the sake of conciseness. 


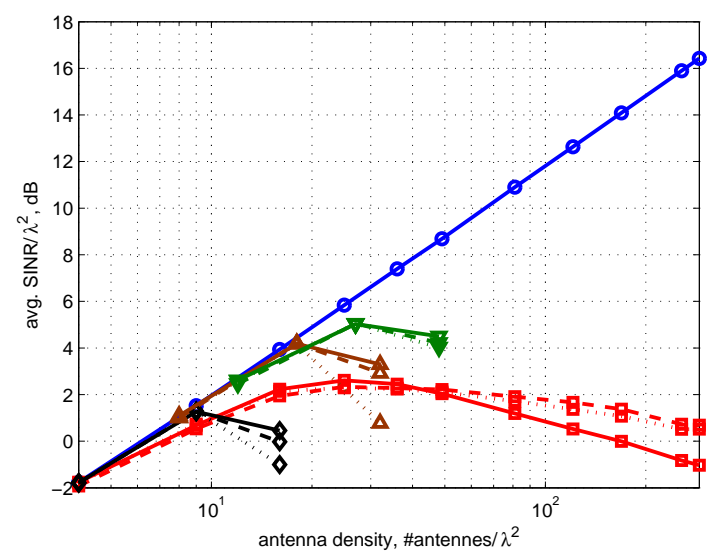

Fig. 4. Comparison of different matching conditions.(-):input impedance ( - - ):self-impedance ( : : ):characteristic impedance $(\circ)$ : ideal antennas $(\square)$ : dipoles $(\diamond)$ : single-polarized patches $(\triangle)$ : dual-polarized patches $(\nabla)$ : tri-polarized radiator

It can be observed that for patch-based elements, the three matching conditions provide similar performances below the optimum point, since the mutual coupling is still quite low. Beyond this point, the input impedance match outperforms the other two since it takes in account also the mutual-impedances. In the dipoles case, the optimum point presents higher mutual coupling thus, the input impedance match is found to be better than the other two techniques. However, it is interesting to highlight that as the antenna density is increased beyond the optimum point, the input impedance match provides the worse results since most of the power injected to the antennas is coupled to other antennas and not radiated towards the users.

\section{E. Microstrip Patches with Improved Bandwidth}

In this section we present two designs of microstrip patches with improved bandwidths since, as mentioned in Section III-B, base station antennas may require in practice bandwidths larger than those achieved by previous patch solutions

The first approach proposed simply consists in increasing the substrate thickness [28]. Here we used a $0.8 \mathrm{~mm}$-thick substrate with $\epsilon_{r}=2.2$ placed $5 \mathrm{~mm}$ above the ground plane, thereby constituting an effectively thick ground plane. The lower permittivity of the substrate implies that the patch must be enlarged to preserve the operation frequency of $2.5 \mathrm{GHz}$, as detailed in Table I. Besides, the long feeding pin of the coaxial probe results in inductive input impedance due to the long feeding pin that has been compensated by cutting rectangular capacitive slots 


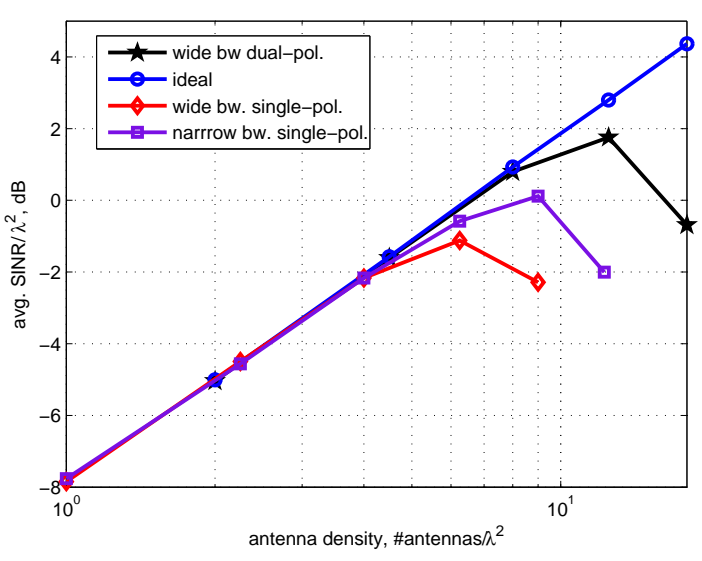

(a) Thick-substrate patch element (See Fig 1(c)).

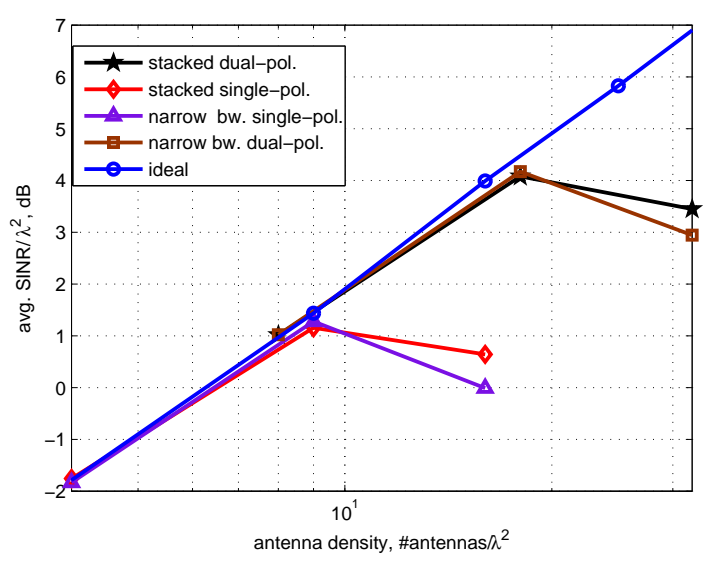

(b) Stacked patch element (See Fig 1(d)).

Fig. 5. SINR evaluation of the bandwidth-improved elements, in comparison with single-layer implementation.

in the patch surrounding the feeding. points, as depicted in Fig. 1(c). Single and dual-polarized elements were designed, achieving $6 \mathrm{~dB}$ bandwidths of $225 \mathrm{MHz}$ and isolation above $25 \mathrm{~dB}$. The second approach is based on stacking patches as shown in Fig. 1(d) [29]. A 6dB bandwidth of $180 \mathrm{MHz}$ was achieved by placing a parasitic patch $10 \mathrm{~mm}$ above the single or the dual polarized patch designed before. In the dual-polarized case, the isolation between ports is above $-25 \mathrm{~dB}$ across the operating bandwidth. Figure 5(a) compares the performance of indoor Massive-MIMO arrays made of these new elements, with that of the single-polarized patches with thin substrate used before. The MRT in noise-limited regime is considered, and both correlation and efficiency are accounted for. As expected, the dual-polarized array with thick substrate provides a 3dB SINR improvement with respect to the single-polarized. However, as aforementioned the bandwidth improvement comes at the price of larger element size. It is seen in Fig. 5(a) that this translates in a reduction of the antennas density with regard to the thin substrate patches, hence poorer SINRs. In contrast, the wideband stacked element behaves as well as its single-layer narrowband counterpart as far as MIMO gain is concerned since it preserves the original patch size. Of course this comes at the price of a higher antenna profile.

\section{F. Miniaturization and decoupling structures}

In this section we complete the study of compact arrays by briefly discussing the use of miniaturization techniques and decoupling structures. On one hand, a miniaturized dual-polarized 


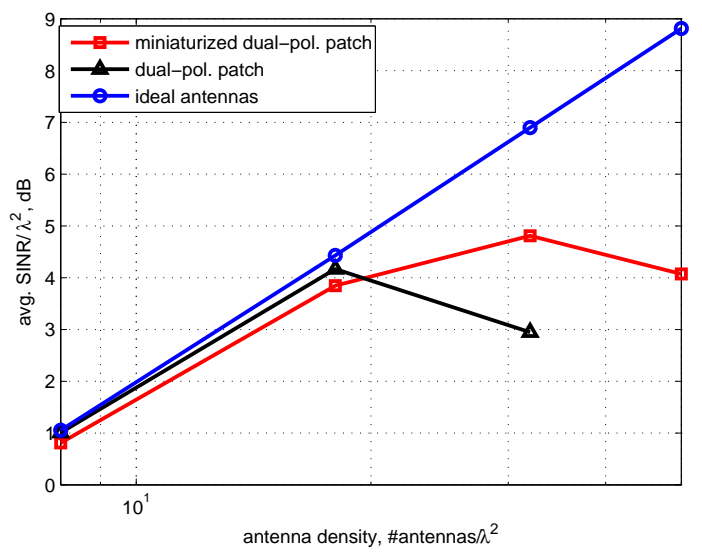

Fig. 6. Patch miniaturization performance evaluation.

patch was designed by cutting four L-shaped slots in the patch following the procedure in [28], as shown in Fig. 1(b) A size reduction of $56 \%$ was achieved with regard to the dual-polarized patch, but with an operating bandwidth decrease of $45 \%$, as shown in Table I. This is a consequence of the well-known antenna limit implying that the product of bandwidth, efficiency and volume must remain constant.

Figure 6 compares the performance achieved with and without patch miniaturization. The size reduction results in the shifting of the optimum point to higher antenna densities. However, the associated improvement in SINR is not significant since the reduced spacing between the miniaturized elements also comes with higher mutual coupling. Therefore, and taking in account that the size reduction necessarily implies bandwidth or efficiency degradation [30], we can conclude that miniaturization is not an effective way to improve Massive-MIMO BS performance.

On the other hand, potential antenna "decoupling" techniques such as electronic band-gap (EBG) [31], etching patterns on the ground plane [32] or metallic shielding between antenna elements were considered. However, it was observed that such techniques are either impossible to implement due to the small space between elements, or are simply ineffective in terms of SINR improvement.

\section{SUMmARY AND Discussion}

A first and extensive discussion on the selection of adequate antenna solutions and interelement spacing for the use of Massive-MIMO technology in indoor scenarios was proposed. 
The main tool employed to this purpose is the computation of the SINR as a function of, on the one hand, antenna type and density (since in Massive-MIMO a very large number of antennas must be accommodated in finite base station real estate)and, on the other hand, the multi-user MIMO precoding strategy.

Firstly, fundamental behavioral observations were made, including the demonstration of the existence of optimal BS antenna density in almost all tested scenarios. It is noticeable that such an optimum was previously identified in single-user MIMO [17], [18] or in Massive-MIMO [11], but using only arrays of dipole antennas through with analytic formulation. We observed here that though an optimum point is observed in the different cases, the achieved SINR and corresponding antenna density significantly varies with the type of element used. For instance, it was shown that though dipoles potentially allow very high antenna densities, this solution is well surpassed by the use of multi-polarized patch antennas in scenarios with XPR close to 0 $\mathrm{dB}$, since they allow both a higher SINR and smaller antenna density, hence better performance at reduced cost. Although this analysis was carried out at a single frequency in order to keep manageable computation times, we briefly addressed the bandwidth issue showing that by using stacked patches large bandwidth can be achieved while preserving MIMO gain.

For the sake of completeness, the effect of antenna elements miniaturization was also evaluated. Interestingly, it was observed that it is not beneficial to Massive-MIMO. Indeed, despite allowing higher antenna density the optimum SINR is not significantly improved (and cost would be higher since this optimum corresponds to a larger antenna density) because coupling remains similar.

A more straightforward issue is associated with the cost incurred by additional antennas at the base station. In practice Massive-MIMO will use near but sub-optimal number of antennas, thereby trading acceptable performance degradation with saving in expensive RF chains. For this purpose the slope of the SINR to density curve around the optimal SINR is obviously determining.

Potential limitations to the generality of these conclusions lie in the assumptions made concerning channel models, the precoders employed, and to the fact that obviously only a finite number of antenna solutions could be tested (the most relevant of which having been reported here). A measured XPR close to $0 \mathrm{~dB}$ and a uniform angular distribution of scatterer's clusters were reported respectively in [21] and [20], thus the assumptions that allowed us to use a simple formulation for the antenna correlation matrices are rather realistic. The linear precod- 
ing techniques considered seem to be the most suitable for Massive-MIMO implementations. Finally, regarding the antenna selection it is reasonable to consider that only marginal variations will be obtained for any other realistic antenna element since different scenarios in terms of volume occupation, multi-polarization, and miniaturization techniques, share the same physical phenomena underlying the existence of an optimal antenna density and associated SINR.

\section{REFERENCES}

[1] G. J. Foschini and M. J. Gans, "On limits of wireless communications in a fading environment when using multiple antennas," Wireless Pers. Commun., vol. 6, pp. 311-335, 1998.

[2] I.E. Telatar, "Capacity of multi-antenna Gaussian channels,” Europ. Trans. Telecommun., vol. 10, pp. 585-595, Nov. 1999.

[3] D. Tse and P. Viswanath, Fundamentals of wireless communication. Cambridge University Press, 2005.

[4] A. Paulraj, R. Nabar, and D. Gore, Introduction to space-time wireless communications. Cambridge University Press, 2003.

[5] D. Gesbert, M. Kountouris, R. Heath, C.-B. Chae, and T. Salzer, "Shifting the mimo paradigm," Signal Processing Magazine, IEEE, vol. 24, no. 5, pp. 36 -46, sept. 2007.

[6] P.Viswanath and D.N.C.Tse, "Sum capacity of the vector Gaussian broadcast channel and uplink-downlink duality," IEEE Trans. Inf. Theory, vol. 49, no. 8, pp. 1912-1921, Aug. 2003.

[7] S. Vishwanath, N. Jindal, and A. Goldsmith, "Duality, achievable rates, and sum-rate capacity of Gaussian MIMO broadcast channels," IEEE Trans. Inf. Theory, vol. 49, no. 10, pp. 2658-2668, Oct. 2003.

[8] T. Marzetta, "How much training is required for multiuser MIMO?" in Signals, Systems and Computers, 2006. ACSSC '06. Fortieth Asilomar Conference on, 29 2006-nov. 1 2006, pp. 359 -363.

[9] —- "Noncooperative cellular wireless with unlimited numbers of base station antennas," Wireless Communications, IEEE Transactions on, vol. 9, no. 11, pp. 3590 -3600, november 2010.

[10] H. Huh, G. Caire, H. Papadopoulos, and S. Ramprashad, "Achieving large spectral efficiency with TDD and not-so-many base-station antennas," in Antennas and Propagation in Wireless Communications (APWC), 2011 IEEE-APS Topical Conference on, sept. 2011, pp. $1346-1349$.

[11] F. Rusek, D. Persson, B. K. Lau, E. G. Larsson, T. L. Marzetta, O. Edfors, and F. Tufvesson, "Scaling up MIMO: Opportunities and challenges with very large arrays," IEEE Signal Processing Magazine, to appear, arXiv:1201.3210.

[12] C. Shepard, H. Yu, N. Anand, E. Li, T. Marzetta, R. Yang, and L. Zhong, "Argos: Practical many-antenna base stations,” in ACM Int. Conf. Mobile Computing and Networking (MobiCom), Aug. 2012.

[13] J. Wallace and M. Jensen, "Mutual coupling in mimo wireless systems: a rigorous network theory analysis," Wireless Communications, IEEE Transactions on, vol. 3, no. 4, pp. 1317 - 1325, july 2004.

[14] T. Svantesson and A. Ranheim, "Mutual coupling effects on the capacity of multielement antenna systems," in Acoustics, Speech, and Signal Processing, 2001. Proceedings. (ICASSP '01). 2001 IEEE International Conference on, vol. 4, 2001, pp. $2485-2488$ vol.4.

[15] C. Waldschmidt, S. Schulteis, and W. Wiesbeck, "Complete rf system model for analysis of compact mimo arrays," Vehicular Technology, IEEE Transactions on, vol. 53, no. 3, pp. 579 - 586, may 2004. 
[16] P. Fletcher, M. Dean, and A. Nix, "Mutual coupling in multi-element array antennas and its influence on mimo channel capacity," Electronics Letters, vol. 39, no. 4, pp. 342 - 344, feb 2003.

[17] R. Janaswamy, "Effect of element mutual coupling on the capacity of fixed length linear arrays," Antennas and Wireless Propagation Letters, IEEE, vol. 1, no. 1, pp. 157 -160, 2002.

[18] S. Shen, M. McKay, and R. Murch, "MIMO systems with mutual coupling: How many antennas to pack into fixed-length arrays?" in Information Theory and its Applications (ISITA), 2010 International Symposium on, oct. 2010, pp. 531 -536.

[19] F. De Flaviis, L. Jofre, J. Romeu, and A. Grau, Multiantenna systems for MIMO comunications. Morgan \& Claypool, 2008.

[20] Q. Spencer, B. Jeffs, M. Jensen, and A. Swindlehurst, "Modeling the statistical time and angle of arrival characteristics of an indoor multipath channel," Selected Areas in Communications, IEEE Journal on, vol. 18, no. 3, pp. 347-360, 2000.

[21] P. Kyritsi, D. Cox, R. Valenzuela, and P. Wolniansky, "Effect of antenna polarization on the capacity of a multiple element system in an indoor environment," Selected Areas in Communications, IEEE Journal on, vol. 20, no. 6, pp. 1227-1239, 2002.

[22] J.-P. Kermoal, L. Schumacher, F. Frederiksen, and P. Mogensen, "Polarization diversity in mimo radio channels: experimental validation of a stochastic model and performance assessment," in Vehicular Technology Conference, 2001. VTC 2001 Fall. IEEE VTS 54th, vol. 1, 2001, pp. 22-26 vol.1.

[23] J. Sarrazin, Y. Mahe, S. Avrillon, and S. Toutain, "Collocated microstrip antennas for MIMO systems with a low mutual coupling using mode confinement," Antennas and Propagation, IEEE Transactions on, vol. 58, no. 2, pp. 589 -592, feb. 2010.

[24] P. Mogensen, T. Koivisto, K. Pedersen, I. Kovacs, B. Raaf, K. Pajukoski, and M. Rinne, "LTE-advanced: The path towards gigabit/s in wireless mobile communications," in Wireless Communication, Vehicular Technology, Information Theory and Aerospace Electronic Systems Technology, 2009. Wireless VITAE 2009. 1st International Conference on, may 2009, pp. $147-151$.

[25] B. K. Lau, J. Andersen, A. Molisch, and G. Kristensson, "Antenna matching for capacity maximization in compact MIMO systems," in Wireless Communication Systems, 2006. ISWCS '06. 3rd International Symposium on, sept. 2006, pp. 253 $-257$.

[26] J. Andersen and B. Lau, "On closely coupled dipoles in a random field," Antennas and Wireless Propagation Letters, IEEE, vol. 5, no. 1 , pp. $73-75$, dec. 2006.

[27] M. Jensen and B. K. Lau, "Uncoupled matching for active and passive impedances of coupled arrays in mimo systems," Antennas and Propagation, IEEE Transactions on, vol. 58, no. 10, pp. 3336 -3343, oct. 2010.

[28] K.-L. Wong, Compact and Broadband Microstrip Antennas. John Wiley \& Sons, 2002.

[29] R. Waterhouse, "Design of probe-fed stacked patches," Antennas and Propagation, IEEE Transactions on, vol. 47, no. 12, pp. $1780-1784$, dec 1999.

[30] C. A. Balanis, Antenna Theory - Analysis and Design. John Wiley \& Sons, 2005.

[31] F. Yang and Y. Rahmat-Samii, "Microstrip antennas integrated with electromagnetic band-gap (EBG) structures: a low mutual coupling design for array applications," Antennas and Propagation, IEEE Transactions on, vol. 51, no. 10, pp. 2936 - 2946, oct. 2003. 
[32] C.-Y. Chiu, C.-H. Cheng, R. Murch, and C. Rowell, "Reduction of mutual coupling between closely-packed antenna elements," Antennas and Propagation, IEEE Transactions on, vol. 55, no. 6, pp. 1732 -1738, june 2007. 